\title{
Monotonicity of a mean related to polygamma functions with an application
}

\section{Zhen-Hang Yang ${ }^{1,2}$ and Shen-Zhou Zheng ${ }^{1 *}$}

\section{"Correspondence:}

shzhzheng@bjtu.edu.cn

'Department of Mathematics,

Beijing Jiaotong University, Beijing,

100044, China

Full list of author information is

available at the end of the article

\begin{abstract}
Let $\psi_{n}=(-1)^{n-1} \psi^{(n)}(n=0,1,2, \ldots)$, where $\psi^{(n)}$ denotes the psi and polygamma functions. We prove that for $n \geq 0$ and two different real numbers $a$ and $b$, the function$$
x \mapsto \psi_{n}^{-1}\left(\frac{\int_{a}^{b} \psi_{n}(x+t) d t}{b-a}\right)-x
$$

is strictly increasing from $(-\min (a, b), \infty)$ onto $(\min (a, b),(a+b) / 2)$, which generalizes a well-known result. As an application, the complete monotonicity for a ratio of gamma functions is improved.
\end{abstract}

\section{Introduction}

The classical Euler's gamma and psi (or called digamma) functions are defined for $x>0$ by

$$
\Gamma(x)=\int_{0}^{\infty} e^{-t} t^{x-1} d t, \quad \psi(x)=\frac{\Gamma^{\prime}(x)}{\Gamma(x)}
$$

respectively. Furthermore, the derivatives $\psi^{\prime}, \psi^{\prime \prime}, \ldots, \psi^{(i)}$ for $i=1,2, \ldots$, are called polygamma functions.

For convenience, we denote $\psi_{n}(x)=(-1)^{n-1} \psi^{(n)}(x)$. It is well known that $\psi_{n}(x)$ is strictly complete monotonic on $(0, \infty)$; namely, $(-1)^{n-1} \psi^{(n)}(x)>0$ for $x>0$ and $n \in \mathbb{N}$. Note that for the following integral and series representations (see [1], Sections 6.3, 6.4):

$$
\begin{aligned}
& \psi_{0}(x)=-\psi(x)=\gamma+\int_{0}^{\infty} \frac{e^{-x t}-e^{-t}}{1-e^{-t}} d t=\gamma+\frac{1}{x}-\sum_{k=1}^{\infty} \frac{x}{k(x+k)}, \\
& \psi_{n}(x)=(-1)^{n-1} \psi^{(n)}(x)=\int_{0}^{\infty} \frac{t^{n}}{1-e^{-t}} e^{-x t} d t=n ! \sum_{k=0}^{\infty} \frac{1}{(x+k)^{n+1}},
\end{aligned}
$$

it is easy to see that $\psi_{n}\left(0^{+}\right)=\infty$ for $n \geq 0, \psi_{n}(\infty)=0$ for $n \geq 1$, and $\psi_{0}(\infty)=-\infty$. Moreover, $\psi_{n}^{\prime}=-\psi_{(n+1)}(x)<0$.

Let $f: I \rightarrow \mathbb{R}$ be strictly monotone and $a, b \in I$. Then the so-called integral $f$-mean of $a$ and $b$ is defined in [2] by

(c) 2016 Yang and Zheng. This article is distributed under the terms of the Creative Commons Attribution 4.0 International License (http://creativecommons.org/licenses/by/4.0/), which permits unrestricted use, distribution, and reproduction in any medium, provided you give appropriate credit to the original author(s) and the source, provide a link to the Creative Commons license, and indicate if changes were made. 


$$
I_{f}(a, b)=f^{-1}\left(\frac{\int_{a}^{b} f(x) d x}{b-a}\right) \quad \text { if } a \neq b, \quad \text { and } \quad I_{f}(a, a)=a \text {. }
$$

For $f=\psi$, Elezović and Pečarić [2], Theorem 6, proved an interesting result as follows.

Theorem EP For $x, a, b>0$, the digamma function $\psi$ has the following properties:

(i) $I_{\psi^{\prime}}(a, b) \leq I_{\psi}(a, b)$; namely,

$$
\left(\psi^{\prime}\right)^{-1}\left(\frac{\int_{a}^{b} \psi^{\prime}(x) d x}{b-a}\right) \leq \psi^{-1}\left(\frac{\int_{a}^{b} \psi(x) d x}{b-a}\right) .
$$

(ii) $x \mapsto I_{\psi}(x+a, x+b)-x$ is increasing concave, and

$$
\lim _{x \rightarrow \infty}\left[I_{\psi}(x+a, x+b)-x\right]=\frac{a+b}{2} .
$$

Remark 1.1 It should be noted that, for $a, b \in I$, if $A(a, b)$ is a mean of $a$ and $b$, then for $x+a, x+b \in I$ the function $x \mapsto A(x+a, x+b)-x$ is still a mean of $a$ and $b$, which is due to the following relations:

$$
\begin{aligned}
\min (a, b) & =\min (x+a, x+b)-x \leq A(x+a, x+b)-x \\
& \leq \max (x+a, x+b)-x=\max (a, b) .
\end{aligned}
$$

Further, Batir [3], Theorem 2.7, gave a nice double inequality for $I_{\psi_{n}}(a, b)$ as follows.

Theorem B Let $a$ and $b$ be distinct positive real numbers and $n$ be a positive integer. Then we have

$$
(-1)^{n} \psi^{(n+1)}\left(\frac{a+b}{2}\right)<(-1)^{n} \frac{\psi^{(n)}(a)-\psi^{(n)}(b)}{a-b}<(-1)^{n} \psi^{(n+1)}\left(S_{-(n+1)}(a, b)\right),
$$

or, equivalently,

$$
S_{-(n+1)}(a, b)<I_{\psi_{n+1}}(a, b)=\psi_{n+1}^{-1}\left(\frac{\int_{a}^{b} \psi_{n+1}(t) d t}{b-a}\right)<\frac{a+b}{2},
$$

where

$$
S_{p}(a, b)= \begin{cases}\left(\frac{a^{p}-b^{p}}{p(a-b)}\right)^{1 /(p-1)}, & \text { if } p \neq 0,1, \\ \frac{a-b}{\ln a-\ln b}, & \text { if } p=0, \\ e^{-1}\left(\frac{a^{a}}{b^{b}}\right)^{1 /(a-b)}, & \text { if } p=1,\end{cases}
$$

is the generalized logarithmic mean of $a$ and $b$.

An improvement of Theorem B was given in [4], Theorem 1, and [5], Theorem 1, by Qi as follows.

Theorem Q1 For real numbers $a, b>0$ with $a \neq b$ and an integer $n \geq 0$, the inequality

$$
(-1)^{n} \psi^{(n)}\left(S_{p}(a, b)\right)<(-1)^{n} \frac{\int_{a}^{b} \psi^{(n)}(t) d t}{b-a} \leq(-1)^{n} \psi^{(n)}\left(S_{q}(a, b)\right)
$$


or

$$
S_{p}(a, b)<I_{\psi_{n}}(a, b)=\psi_{n}^{-1}\left(\frac{\int_{a}^{b} \psi_{n}(t) d t}{b-a}\right) \leq S_{q}(a, b)
$$

holds if $p \leq-n$ and $q \geq-n+1$, where $S_{p}(a, b)$ is given in (1.3).

Motivated by the results just mentioned, the main aim of this paper is to continue the study of some further properties of the mean $I_{\psi_{n}}(a, b)$ and $I_{\psi_{n}}(x+a, x+b)-x$. More precisely, we have the following.

Theorem 1.2 For $a, b>0$ with $a \neq b$, the sequence $\left\{I_{\psi_{n}}(a, b)\right\}_{n \geq 0}$ is strictly decreasing, and

$$
\lim _{n \rightarrow \infty} I_{\psi_{n}}(a, b)=\min (a, b)
$$

Theorem 1.3 Let a and $b$ be distinct real numbers, and $n \geq 0$ be an integer. If $\psi_{n}^{-1}$ is strictly decreasing with respected to $x$, then the function $x \mapsto A_{\psi_{n}}(x)$ with

$$
A_{\psi_{n}}(x)=I_{\psi_{n}}(x+a, x+b)-x=\psi_{n}^{-1}\left(\frac{\int_{a}^{b} \psi_{n}(x+t) d t}{b-a}\right)-x
$$

is strictly increasing from $(-\min (a, b), \infty)$ onto $(\min (a, b),(a+b) / 2)$.

As a direct consequence, noting that $\psi_{n}^{-1}$ is strictly decreasing, by Theorem 1.3 we have the following.

Corollary 1.4 Let $a$ and $b$ be distinct real numbers and $n \geq 0$ be an integer. Then for $x>-\min (a, b)$ we have

$$
\psi_{n}\left(x+\frac{a+b}{2}\right)<\frac{\int_{a}^{b} \psi_{n}(x+t) d t}{b-a}<\psi_{n}(x+\min (a, b)),
$$

where $\min (a, b)$ and $(a+b) / 2$ are the best constants. In particular, note that $\psi_{0}=-\psi$, the double inequality

$$
\psi(x+\min (a, b))<\frac{\int_{a}^{b} \psi(x+t) d t}{b-a}<\psi\left(x+\frac{a+b}{2}\right)
$$

or

$$
\exp \psi(x+\min (a, b))<\left[\frac{\Gamma(x+b)}{\Gamma(x+a)}\right]^{1 /(b-a)}<\exp \psi\left(x+\frac{a+b}{2}\right)
$$

holds for $x>-\min (a, b)$ with the best constants $\min (a, b)$ and $(a+b) / 2$.

Suppose that $a, b>0$ with $a \neq b$ in Theorem 1.3 . Utilizing the strictly increasing property of $x \mapsto A_{\psi_{n}}(x)$ on $(0, \infty)$, we have $A_{\psi_{n}}(0)<A_{\psi_{n}}(x)<A_{\psi_{n}}(\infty)$; namely,

$$
I_{\psi_{n}}(a, b)=\psi_{n}^{-1}\left(\frac{\int_{a}^{b} \psi_{n}(t) d t}{b-a}\right)<\psi_{n}^{-1}\left(\frac{\int_{a}^{b} \psi_{n}(x+t) d t}{b-a}\right)-x<\frac{a+b}{2} .
$$

Therefore, we conclude the following. 
Corollary 1.5 Let $a, b>0$ with $a \neq b$ and $n \geq 0$ be an integer. Then for $x>0$ we have

$$
\psi_{n}\left(x+\frac{a+b}{2}\right)<\frac{\int_{a}^{b} \psi_{n}(x+t) d t}{b-a}<\psi_{n}\left(x+I_{\psi_{n}}(a, b)\right)
$$

where $I_{\psi_{n}}(a, b)$ and $(a+b) / 2$ are the best constants. Particularly, noting that $\psi_{0}=-\psi$, the double inequality

$$
\psi\left(x+I_{\psi}(a, b)\right)<\frac{\int_{a}^{b} \psi(x+t) d t}{b-a}<\psi\left(x+\frac{a+b}{2}\right)
$$

or

$$
\exp \psi\left(x+I_{\psi}(a, b)\right)<\left[\frac{\Gamma(x+b)}{\Gamma(x+a)}\right]^{1 /(b-a)}<\exp \psi\left(x+\frac{a+b}{2}\right)
$$

holds for $x>0$ with the best constants $I_{\psi}(a, b)$ and $(a+b) / 2$.

We would think it worth noticing that the double inequality (1.6) was first proved in [6] by Elezović et al.

Remark 1.6 The second Kershaw double inequality [7] states that

$$
\exp [(1-s) \psi(x+\sqrt{s})]<\frac{\Gamma(x+1)}{\Gamma(x+s)}<\exp \left[(1-s) \psi\left(x+\frac{s+1}{2}\right)\right]
$$

for $s \in(0,1)$ and $x \geq 0$. Some of the refinements, extensions, and generalizations of the double inequality (1.7) can be found in Qi's review paper [8] and the references therein. It seems that our double inequality (1.5) may be the best second Kershaw type inequality, since the ranges of $a$ and $b$ in (1.5) are arbitrary real numbers, and the lower and upper bounds are sharp.

As an application of Theorem 1.3, we use it to prove a necessary and sufficient condition for the functions $x \mapsto F_{a, b, c}(x)$ defined by (3.1) and $x \mapsto 1 / F_{a, b, c}(x)$ to be logarithmically monotonic on $(-\rho, \infty)$ with $\rho=\min (a, b, c)$, which improves a well-known result.

\section{Proofs of main results}

This section we devote to the proof of our main results. First of all, let us give the following assertion, which is an improvement of Theorem 4 in [2].

Lemma 2.1 Let $f \in C^{(2)}(I)$. Iff is strictly monotone, then the mean function

$$
A_{f}(x)=I_{f}(a+x, b+x)-x=f^{-1}\left(\frac{\int_{a}^{b} f(x+t) d t}{b-a}\right)-x
$$

is strictly increasing (decreasing) according to $f^{\prime \prime} \mid f^{\prime}$ being strictly increasing (decreasing). 
Proof By the Jensen inequality we have

$$
f^{\prime}\left(f^{-1}\left(\frac{\int_{a}^{b} f(x+t) d t}{b-a}\right)\right)<(>) \frac{\int_{a}^{b} f^{\prime}(x+t) d t}{b-a}
$$

if $f^{\prime} \circ f^{-1}$ is strictly convex (concave).

Differentiation yields

$$
\frac{d f^{\prime}\left(f^{-1}(x)\right)}{d x}=f^{\prime \prime}\left(f^{-1}(x)\right) \frac{d\left(f^{-1}(x)\right)}{d x}=\frac{f^{\prime \prime}\left(f^{-1}(x)\right)}{f^{\prime}\left(f^{-1}(x)\right)}=\frac{f^{\prime \prime}(u)}{f^{\prime}(u)},
$$

where $u=f^{-1}(x)$. This shows that $f^{\prime} \circ f^{-1}$ is strictly convex if and only if both $f$ and $f^{\prime \prime} / f^{\prime}$ are either increasing or decreasing, and concave if and only if one of $f$ and $f^{\prime \prime} / f^{\prime}$ is increasing, while the other is decreasing.

Case 1: Both $f$ and $f^{\prime \prime} / f^{\prime}$ are increasing. Then $f^{\prime}>0$ and $f^{\prime} \circ f^{-1}$ is convex, and it follows from (2.2) that

$$
\frac{d A_{f}(x)}{d x}=\frac{\int_{a}^{b} f^{\prime}(x+t) d t}{b-a} / f^{\prime}\left(f^{-1}\left(\frac{\int_{a}^{b} f(x+t) d t}{b-a}\right)\right)-1>0 .
$$

Case 2: $f$ is decreasing and $f^{\prime \prime} / f^{\prime}$ is increasing. Then $f^{\prime}<0$ and $f^{\prime} \circ f^{-1}$ is concave and by (2.2) we also have $d A_{f}(t) / d t>0$.

Case 3: Both $f$ and $f^{\prime \prime} / f^{\prime}$ are decreasing. Then $f^{\prime}<0$ and $f^{\prime} \circ f^{-1}$ is convex. Similarly, we have $d A_{f}(t) / d t<0$.

Case 4: $f$ is increasing and $f^{\prime \prime} / f^{\prime}$ is decreasing. Then $f^{\prime}>0$ and $f^{\prime} \circ f^{-1}$ is concave. Obviously, we see that $d A_{f}(t) / d t<0$.

To sum up, if $f^{\prime \prime} / f^{\prime}$ is increasing (decreasing), then so is $A_{f}$, which completes the proof.

The following lemma is useful for our main proof, which is a generalization of Lemma 1.4 in [3] and Lemma 4 in [9].

Lemma 2.2 Let $A:(0, \infty) \times(0, \infty) \rightarrow(0, \infty)$ be a differentiable one-order homogeneous mean. Then, for all $x+t, y+t \in(0, \infty)$, we have

$$
\lim _{t \rightarrow \infty}(A(x+t, y+t)-t)=p x+(1-p) y
$$

where $p=A_{x}(1,1) \in[0,1]$. In particular, if $A(x, y)$ is symmetric with respect to $x$ and $y$, then

$$
\lim _{t \rightarrow \infty}(A(x+t, y+t)-t)=\frac{x+y}{2} .
$$

Proof Using homogeneity of $A(x, y)$ and the L'Hospital rule yield

$$
\begin{aligned}
\lim _{t \rightarrow \infty}(A(x+t, y+t)-t) & =\lim _{t \rightarrow \infty} \frac{A\left(t^{-1} x+1, t^{-1} y+1\right)-1}{t^{-1}} \\
& \stackrel{t^{-1}=u}{=} \lim _{u \rightarrow 0} \frac{A(u x+1, u y+1)-1}{u} \\
& =\lim _{u \rightarrow 0} \frac{\partial A(u x+1, u y+1)}{\partial u}=x A_{x}(1,1)+y A_{y}(1,1) .
\end{aligned}
$$


In addition, it follows from [10] that

$$
A_{x}(x, x), A_{y}(x, x) \in[0,1] \quad \text { and } \quad A_{x}(x, x)+A_{y}(x, x)=1 .
$$

Putting the above together, we get (2.3).

In particular, if $A$ is symmetric, that is, $A(x, y)=A(y, x)$, then we clearly see that $A_{x}(x, y)=$ $A_{y}(y, x)$, and so $A_{x}(x, x)=A_{y}(x, x)$. It follows from $(2.5)$ that $A_{x}(x, x)=A_{y}(x, x)=1 / 2$, and then (2.4) holds. The proof is complete.

Lemma 2.3 Let $\psi_{n}=(-1)^{n-1} \psi^{(n)}$ for $n \in \mathbb{N}$. Then all the following statements are true, and mutually equivalent.

(i) the sequence $\left\{\psi_{n+1} / \psi_{n}\right\}_{n \in \mathbb{N}}$ is strictly increasing;

(ii) the function $x \mapsto \psi_{n+1}(x) / \psi_{n}(x)$ is strictly decreasing on $(0, \infty)$;

(iii) the function $x \mapsto \psi_{n}(x)$ is log-convex on $(0, \infty)$.

Proof (i) It suffices to prove $\psi_{n+2} / \psi_{n+1}>\psi_{n+1} / \psi_{n}$ for $n \in \mathbb{N}$, which is equivalent to $\psi_{n+2} \psi_{n}-$ $\psi_{n+1}^{2}>0$. By virtue of the integral representation given in (1.2), we get

$$
\begin{aligned}
\psi_{n+2} \psi_{n}-\psi_{n+1}^{2} & =\int_{0}^{\infty} \frac{t^{n+2}}{1-e^{-t}} e^{-x t} d t \int_{0}^{\infty} \frac{t^{n}}{1-e^{-t}} e^{-x t} d t-\left(\int_{0}^{\infty} \frac{t^{n+1}}{1-e^{-t}} e^{-x t} d t\right)^{2} \\
& =\frac{1}{2} \int_{0}^{\infty} \int_{0}^{\infty} \frac{t^{n} s^{n}(t-s)^{2}}{\left(1-e^{-t}\right)\left(1-e^{-s}\right)} e^{-x(t+s)} d t d s>0,
\end{aligned}
$$

which proves assertion (i).

(ii) Note that $\psi_{n}^{\prime}=-\psi_{n+1}$, we have

$$
\left(\frac{\psi_{n+1}}{\psi_{n}}\right)^{\prime}=\frac{\psi_{n+1}^{\prime} \psi_{n}-\psi_{n+1} \psi_{n}^{\prime}}{\psi_{n}^{2}}=\frac{-\psi_{n+2} \psi_{n}+\psi_{n+1}^{2}}{\psi_{n}^{2}}<0
$$

which implies that the second assertion is true.

(iii) Differentiation gives

$$
\left(\ln \psi_{n}\right)^{\prime}=\frac{\psi_{n}^{\prime}}{\psi_{n}}=-\frac{\psi_{n+1}}{\psi_{n}}, \quad\left(\ln \psi_{n}\right)^{\prime \prime}=-\left(\frac{\psi_{n+1}}{\psi_{n}}\right)^{\prime}>0,
$$

which completes the proof.

Now we are in a position to prove our main results.

Proof of Theorem 1.2 We first prove that the sequence $\left\{I_{\psi_{n}}(a, b)\right\}_{n \geq 0}$ is strictly decreasing, which means that for $n \geq 0$ the inequality

$$
\psi_{n}^{-1}\left(\frac{\int_{a}^{b} \psi_{n}(x) d x}{b-a}\right)>\psi_{n+1}^{-1}\left(\frac{\int_{a}^{b} \psi_{n+1}(x) d x}{b-a}\right)
$$

holds for $a, b>0$ with $a \neq b$. By the Jensen inequality, it suffices to check that $\psi_{n+1} \circ \psi_{n}^{-1}$ is convex on $(0, \infty)$. In fact, by Lemma 2.3 we have

$$
\frac{d}{d x} \psi_{n+1}\left(\psi_{n}^{-1}(x)\right)=\frac{\psi_{n+1}^{\prime}\left(\psi_{n}^{-1}(x)\right)}{\psi_{n}^{\prime}\left(\psi_{n}^{-1}(x)\right)}=\frac{\psi_{n+2}\left(\psi_{n}^{-1}(x)\right)}{\psi_{n+1}\left(\psi_{n}^{-1}(x)\right)}
$$




$$
\frac{d^{2}}{d x^{2}} \psi_{n+1}\left(\psi_{n}^{-1}(x)\right)=\left(\frac{\psi_{n+2}(u)}{\psi_{n+1}(u)}\right)^{\prime} \frac{1}{\psi_{n}^{\prime}(u)}=-\left(\frac{\psi_{n+2}(u)}{\psi_{n+1}(u)}\right)^{\prime} \frac{1}{\psi_{n+1}(u)}>0
$$

where $u=\psi_{n}^{-1}(x)$. This means that $\psi_{n+1} \circ \psi_{n}^{-1}$ is convex, which proves inequality (2.6).

Taking $p=-n$ and $q=-n+1$ in Theorem Q1 gives

$$
S_{-n}(a, b)<\psi_{n}^{-1}\left(\frac{\int_{a}^{b} \psi_{n}(t) d t}{b-a}\right)<S_{-n+1}(a, b)
$$

Considering that $\lim _{p \rightarrow-\infty} S_{p}(a, b)=\min (a, b)$ in [11], then we get

$$
\lim _{n \rightarrow \infty} \psi_{n}^{-1}\left(\frac{\int_{a}^{b} \psi_{n}(t) d t}{b-a}\right)=\min (a, b)
$$

which completes the proof.

Proof of Theorem 1.3 To prove $x \mapsto A_{\psi_{n}}(x)$ is strictly increasing on $(-\min (a, b), \infty)$, by Lemma 2.1 it suffices to check that $\psi_{n}^{\prime \prime} / \psi_{n}^{\prime}$ is strictly increasing on $(0, \infty)$. In fact, since $\psi_{n}^{\prime}=-\psi_{n+1}$ we see that $\psi_{n}^{\prime \prime} / \psi_{n}^{\prime}=-\psi_{n+2} / \psi_{n+1}$ is strictly increasing on $(0, \infty)$ by the second assertion of Lemma 2.3. Thus, the increasing property of $A_{\psi_{n}}$ follows.

As mentioned in the introduction, we see that $\psi_{n}\left(0^{+}\right)=\infty$ for $n \geq 0$, and so $\psi_{n}^{-1}(\infty)=0$. Note that the symmetry of $a$ and $b$, without loss of generality we may assume that $b>a$.

Then we have

$$
\lim _{x \rightarrow-a^{+}} \frac{\int_{a}^{b} \psi_{n}(x+t) d t}{b-a}=\lim _{x \rightarrow-a^{+}} \frac{(-1)^{n-1}\left(\psi^{(n-1)}(x+b)-\psi^{(n-1)}(x+a)\right)}{b-a}=\infty,
$$

which implies

$$
\begin{aligned}
\lim _{x \rightarrow-a^{+}} A_{\psi_{n}}(x) & =\lim _{x \rightarrow-a^{+}} \psi_{n}^{-1}\left(\frac{\int_{a}^{b} \psi_{n}(x+t) d t}{b-a}\right)-\lim _{x \rightarrow-a^{+}} x \\
& =\psi_{n}^{-1}\left(\lim _{x \rightarrow-a^{+}} \frac{\int_{a}^{b} \psi_{n}(x+t) d t}{b-a}\right)+a=\psi_{n}^{-1}(\infty)+a=a .
\end{aligned}
$$

To obtain $\lim _{x \rightarrow \infty} A_{\psi_{n}}(x)=(a+b) / 2$, we use (2.7) to derive that

$$
S_{-n}(x+b, x+a)-x<\psi_{n}^{-1}\left(\frac{\int_{a}^{b} \psi_{n}(x+t) d t}{b-a}\right)-x<S_{-n+1}(x+b, x+a)-x
$$

Note that the generalized logarithmic mean $S_{p}(x, y)$ is homogeneous and symmetric, it follows from Lemma 2.2 that

$$
\lim _{x \rightarrow \infty}\left(S_{p}(x+b, x+a)-x\right)=\frac{a+b}{2} .
$$

Therefore, we conclude that $\lim _{x \rightarrow \infty} A_{\psi_{n}}(x)=(a+b) / 2$, which completes the proof. 


\section{An application}

A function $f$ is said to be completely monotonic on an interval $I$ if $f$ has derivatives of all orders on $I$ and $(-1)^{n}(f(x))^{(n)} \geq 0$ for $x \in I$ and $n \geq 0$ (see [12]). A positive function $f$ is called logarithmically completely monotonic on an interval $I$ if $f$ has derivatives of all orders on $I$ and its $\operatorname{logarithm} \ln f$ satisfies $(-1)^{n}(\ln f(x))^{(n)} \geq 0$ for all $n \in \mathbb{N}$ on $I$ (see [13]). For convenience, we denote the sets of the completely monotonic functions and the logarithmically completely monotonic functions on $I$ by $\mathcal{C}[I]$ and $\mathcal{L}[I]$, respectively. Qi in [14], Theorem 1, [15], Theorem 1, investigated the logarithmically complete monotonicity of the functions

$$
x \mapsto F_{a, b, c}(x)= \begin{cases}\left(\frac{\Gamma(x+b)}{\Gamma(x)+a)}\right)^{1 /(a-b)} e^{\psi(x+c),} & \text { if } a \neq b, \\ e^{\psi(x+c)-\psi(x+a),} & \text { if } a=b,\end{cases}
$$

and $x \mapsto 1 / F_{a, b, c}(x)$. Furthermore, he concluded the following result.

Theorem Q2 Let $a, b$, and $c$ be real numbers and $\rho=\min (a, b, c)$. If $\theta(t)$ is an implicit function defined by

$$
e^{t}-t=e^{\theta(t)}-\theta(t)
$$

on $(-\infty, \infty)$, then $\theta(t)$ is decreasing and $t \theta(t)<0$ for $\theta(t) \neq t$. Moreover:

(1) $F_{a, b, c}(x) \in \mathcal{L}[(-\rho, \infty)]$ if

$$
\begin{gathered}
(a, b, c) \in\{c \geq a, c \geq b\} \cup\{c \geq a, 0 \geq c-b \geq \theta(c-a)\} \\
\cup\{c \leq a, c-b \geq \theta(c-a)\} \backslash\{a=b=c\} .
\end{gathered}
$$

(2) $1 / F_{a, b, c}(x) \in \mathcal{L}[(-\rho, \infty)]$ if

$$
\begin{aligned}
(a, b, c) \in\{c \leq a, c \leq b\} \cup\{c \geq a, c-b \leq \theta(c-a)\} \\
\cup\{c \leq a, 0 \leq c-b \leq \theta(c-a)\} \backslash\{a=b=c\} .
\end{aligned}
$$

Later, Qi and Guo in [16], Theorem 1, [17], Theorem 1, proved another result concerning the logarithmically complete monotonicity of the functions $x \mapsto F_{a, b, c}(x)$ and $x \mapsto 1 / F_{a, b, c}(x)$ for $x>-\min (a, b, c)$, where $c=c(a, b)$ is a constant depending on $a$ and $b$. More precisely, they showed the following.

Theorem QG Let $a$ and $b$ be two real numbers with $a \neq b$ and $c(a, b)$ be $a$ constant depending on $a$ and $b$.

(1) If $c(a, b) \leq \min (a, b)$, then $1 / F_{a, b, c}(x) \in \mathcal{L}[(-c(a, b), \infty)]$.

(2) $F_{a, b, c}(x) \in \mathcal{L}[(-\min (a, b), \infty)]$ if and only if $c(a, b) \geq(a+b) / 2$.

We would like to remark that the result in Theorem Q2 is rather interesting but somewhat complicated. Theorem QG shows that $c$ is a constant depending on $a$ and $b$, and $c(a, b) \leq \min (a, b)$ is only sufficient for $1 / F_{a, b, c}(x) \in \mathcal{L}[(-c(a, b), \infty)]$. Here, we apply Theorem 1.3 to deduce that $c$ is a constant independent of $a$ and $b$, and $c \leq \min (a, b)$ is also necessary for $1 / F_{a, b, c}(x) \in \mathcal{L}[(-c(a, b), \infty)]$. This improved result can be restated as follows. 
Theorem 3.1 Let $a, b$, and $c$ be real numbers, and $\rho=\min (a, b, c)$. Then $1 / F_{a, b, c}(x) \in$ $\mathcal{L}[(-\rho, \infty)]$ if and only if $c \leq \min (a, b)$, while $F_{a, b, c}(x) \in \mathcal{L}[(-\rho, \infty)]$ if and only if $c \geq$ $(a+b) / 2$.

Proof For $a \neq b$, we have

$$
\ln F_{a, b, c}(x)=\psi(x+c)-\frac{\ln \Gamma(x+b)-\ln \Gamma(x+a)}{b-a}=\psi(x+c)-\frac{\int_{a}^{b} \psi(x+t) d t}{b-a}
$$

and

$$
\begin{aligned}
(-1)^{n}\left(\ln F_{a, b, c}(x)\right)^{(n)} & =(-1)^{n} \psi^{(n)}(x+c)-\frac{(-1)^{n} \int_{a}^{b} \psi^{(n)}(x+t) d t}{b-a} \\
& =\frac{\int_{a}^{b} \psi_{n}(x+t) d t}{b-a}-\psi_{n}(x+c) \\
& =\frac{(b-a)^{-1} \int_{a}^{b} \psi_{n}(x+t) d t-\psi_{n}(x+c)}{\psi_{n}^{-1}\left((b-a)^{-1} \int_{a}^{b} \psi_{n}(x+t) d t\right)-(x+c)}\left(A_{\psi_{n}}(x)-c\right),
\end{aligned}
$$

where $\psi_{n}=(-1)^{n-1} \psi^{(n)}$ and $A_{\psi_{n}}(x)$ is defined by (1.4).

Since $\psi_{n}^{\prime}=-\psi^{(n+1)}<0,\left(\psi_{n}^{-1}\right)^{\prime}<0$, which means that $\psi_{n}^{-1}$ is strictly decreasing on $(0, \infty)$. This yields

$$
\frac{(b-a)^{-1} \int_{a}^{b} \psi_{n}(x+t) d t-\psi_{n}(x+c)}{\psi_{n}^{-1}\left((b-a)^{-1} \int_{a}^{b} \psi_{n}(x+t) d t\right)-\psi_{n}^{-1}\left(\psi_{n}(x+c)\right)}<0
$$

for $x \in(-\rho, \infty)$. Therefore, we have

$$
\operatorname{sgn}\left((-1)^{n}\left(\ln F_{a, b, c}(x)\right)^{(n)}\right)=\operatorname{sgn}\left(c-A_{\psi_{n}}(x)\right)
$$

Theorem 1.3 tells that $x \mapsto A_{\psi_{n}}(x)=I_{\psi_{n}}(x+a, x+b)-x$ is strictly increasing from $(-\min (a, b), \infty)$ onto $(\min (a, b),(a+b) / 2)$, which implies that

$$
\operatorname{sgn}\left(c-A_{\psi_{n}}(x)\right) \leq 0 \quad \Longleftrightarrow \quad c \leq \inf A_{\psi_{n}}(x)=\min (a, b)
$$

and

$$
\operatorname{sgn}\left(c-A_{\psi_{n}}(x)\right) \geq 0 \quad \Longleftrightarrow \quad c \geq \sup A_{\psi_{n}}(x)=\frac{a+b}{2} .
$$

It is obvious that these are also true for $a=b$. This completes the proof. 
Funding

This paper is partially supported by the National Natural Science Foundation of China with grant No. 11371050.

Received: 12 July 2016 Accepted: 26 August 2016 Published online: 09 September 2016

\section{References}

1. Abramowitz, M, Stegun, IA (eds.): Handbook of Mathematical Functions with Formulas, Graphs, and Mathematical Tables, 10th printing edn. National Bureau of Standards Applied Mathematics Series, vol. 55. Dover, New York (1972)

2. Elezović, N, Pečarić, J: Differential and integral $f$-means and applications to digamma functions. Math. Inequal. Appl. 3(2), 189-196 (2000)

3. Batir, N: On some properties of digamma and polygamma functions. J. Math. Anal. Appl. 328(1), 452-465 (2007)

4. Qi, F: Refinements, extensions and generalizations of the second Kershaw's double inequality. RGMIA Res. Rep. Collect. 10(2), 8 (2007)

5. Qi, F, Li, X-A, Chen, S-X: Refinements, extensions and generalizations of the second Kershaw's double inequality. Math Inequal. Appl. 11(3), 457-465 (2008)

6. Elezović, N, Giordano, C, Pečarić, J: The best bounds in Gautschi's inequality. Math. Inequal. Appl. 3(2), 239-252 (2000)

7. Kershaw, D: Some extensions of W. Gautschi's inequalities for the gamma function. Math. Comput. 41(164), 607-611 (1983)

8. Qi, F: Bounds for the ratio of two gamma functions. J. Inequal. Appl. 2010, Article ID 493058 (2010)

9. Czinder, P, Pales, Z: A general Minkowski-type inequality for two variables Gini means. Publ. Math. (Debr.) 57(1-2), 203-216 (2000)

10. Toader, S: Derivatives of generalized means. Math. Inequal. Appl. 5, 517-532 (2002)

11. Stolarsky, KB: Generalizations of the logarithmic mean. Math. Mag. 48, $87-92$ (1975)

12. Wendel, J: Note on the gamma function. Am. Math. Mon. 55, 563-564 (1948)

13. Qi, F, Chen, C-P: A complete monotonicity property of the gamma function. J. Math. Anal. Appl. 296(2), 603-607 (2004)

14. Qi, F: A class of logarithmically completely monotonic functions and application to the best bounds in the second Gautschi-Kershaw's inequality. J. Comput. Appl. Math. 224(2), 538-543 (2009)

15. Qi, F: A class of logarithmically completely monotonic functions and application to the best bounds in the second Gautschi-Kershaw's inequality. RGMIA Res. Rep. Collect. 9(4), 11 (2006)

16. Qi, F, Guo, B-N: A class of logarithmically completely monotonic functions and the best bounds in the second Kershaw's double inequality. J. Comput. Appl. Math. 212(2), 444-456 (2008)

17. Qi, F, Guo, B-N: A class of logarithmically completely monotonic functions and the best bounds in the second Kershaw's double inequality. RGMIA Res. Rep. Collect. 10(2), 5 (2007)

\section{Submit your manuscript to a SpringerOpen ${ }^{\odot}$ journal and benefit from:}

- Convenient online submission

- Rigorous peer review

- Immediate publication on acceptance

Open access: articles freely available online

- High visibility within the field

- Retaining the copyright to your article 